\title{
A generalization of Apollonian packing of circles
}

\author{
Gerhard Guettler and Colin Mallows
}

\begin{abstract}
Three circles touching one another at distinct points form two curvilinear triangles. Into one of these we can pack three new circles, touching each other, with each new circle touching two of the original circles. In such a sextuple of circles there are three pairs of circles, with each of the circles in a pair touching all four circles in the other two pairs. Repeating the construction in each curvilinear triangle that is formed results in a generalized Apollonian packing. We can invert the whole packing in every circle in it, getting a "generalized Apollonian super-packing". Many of the properties of the Descartes configuration and the standard Apollonian packing carry over to this case. In particular, there is an equation of degree 2 connecting the bends (curvatures) of a sextuple; all the bends can be integers; and if they are, the packing can be placed in the plane so that for each circle with bend $b$ and center $(x, y)$, the quantities $b x / \sqrt{2}$ and $b y$ are integers. The construction provides a generalization of the Farey series and the associated Ford circles.
\end{abstract}

\section{Introduction and summary}

Recently there has been renewed interest in a very old idea, that of Apollonian packing of circles, in which an initial configuration of three mutually tangent circles is augmented by repeatedly drawing new circles in each curvilinear gap. See for example Mumford et al [10]. We can also study "superApollonian" packings which are obtained by repeatedly inverting an Apollonian packing in every circle in it. It is a remarkable fact that Apollonian and super-Apollonian packings exist in which all the bends (curvatures) are integers. This property, and the associated group theory, has been studied in detail by Graham et al [4-7]. Also, if all the bends are integers, the superApollonian packing can be placed in the plane so that all the "bend times center" quantities are integers. Several extensions of the Apollonian idea have been studied, for example Mauldon [9] studied configurations in which adjacent circles do not touch but have constant "separation". Stephenson [12] takes the theory in rather different directions.

Our own interest lies in extending these ideas in new directions, particularly by packing not one but three circles within each triangular gap, thus 


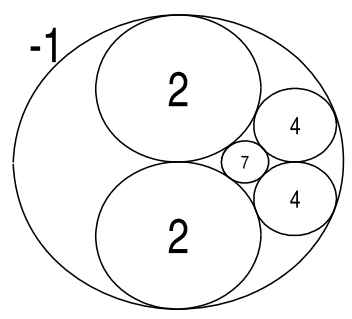

(a)

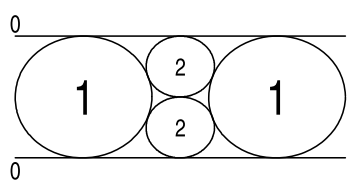

(c)

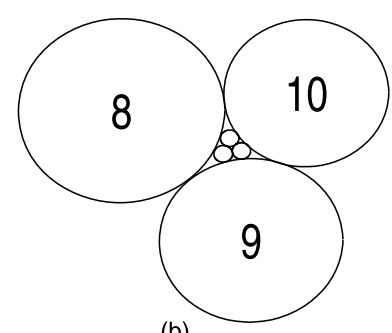

(b)

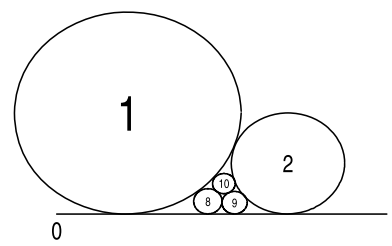

(d)

Figure 1: Sextuple configurations.

forming sextuples of circles, and in exploring the degree to which the theory associated with the classical packings can be extended to cover this case. We find that all the bends in such a generalized packing can be integers; and there are results relating to the positions of the centers of the circles that directly generalize those found by Lagarias et al [8] in the classical Descartes-Apollonian case.

Into either of the curvilinear triangles formed by three mutually tangent circles we can pack three more circles, forming a sextuple. Figure 1 shows the four possible configurations of a sextuple. There can be zero, one, or two circles with bend zero (i.e. straight lines), and at most one bend can be negative, as in case (a).

The circles are labelled with their bends. The bends of the three small circles in Figure 1(b) are 88, 89, and 90.

These generalize the classical Descartes configuration, in which just one circle is placed in a curvilinear triangle. Such a sextuple of circles forms an $n=4$ example of what we call a "ball-bearing" configuration, in which a ring of $n$ circles (each touching two others) have the property that there are "inner" and "outer" circles that each touch all $n$ circles in the ring. The $n=3$ case contains the classical Descartes 4 -circle configuration. With 


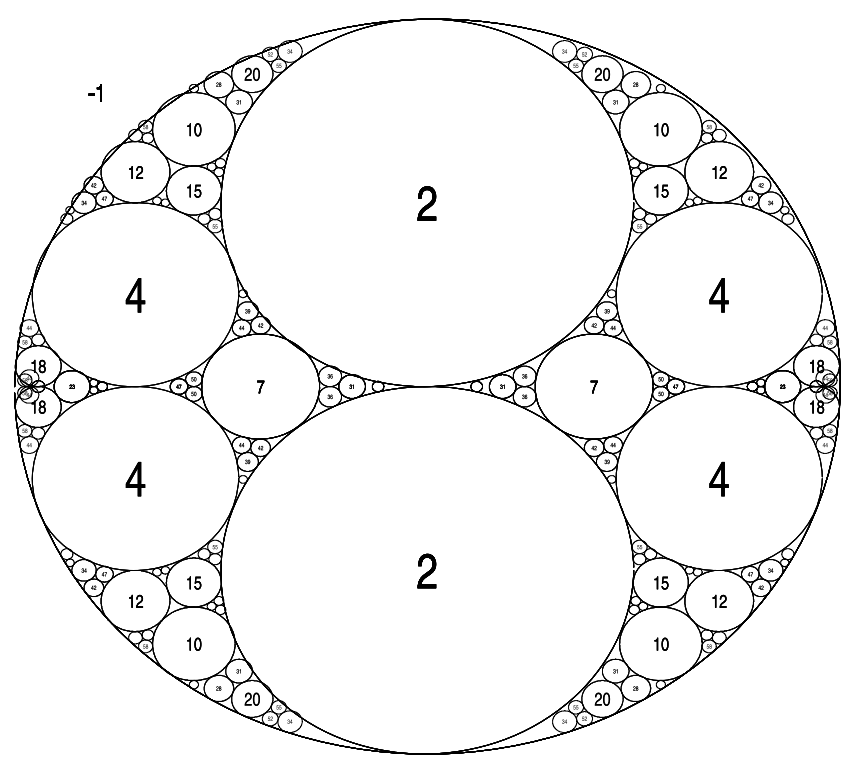

Figure 2: A generalized Apollonian packing.

$n=4$ the circles come in three pairs, with each of the circles in a pair touching all four circles in the other two pairs. The circles of a pair do not touch one another. The sextuple thus has the symmetry of the vertices of an octahedron (or of the faces of a cube), rather than the symmetry of a tetrahedron as in the Descartes case. We describe the bends in a sextuple by a vector of the form $\left(a, a^{\prime} ; b, b^{\prime} ; c, c^{\prime}\right)$. Thus in Figure $1(\mathrm{a})$ the bends are $(-1,7 ; 2,4 ; 2,4)$ and in Figure $1(\mathrm{c})$ they are $(0,2 ; 0,2 ; 1,1)$.

Repeating the construction in each curvilinear triangle that is formed results in a "generalized Apollonian packing". See Figures 2 (based on Figure 1(a)) and 3 (based on Figure 1(c)). In these packings, all the bends are integers. In this and subsequent figures, we have drawn only the circles with bends less than 100. There are also packings that fill either a half-plane, or the whole plane; but their bends cannot all be integral.

Many of the properties of the Descartes configuration and the standard Apollonian packing carry over to this case. In later sections we will establish these results:

(i) Given three mutually tangent circles, there is a quadratic equation ((5) below) whose coefficients involve the bends of these circles, and whose roots determine the bends of the two sets of three circles that can be inscribed in the curvilinear triangles that they form, thus making two sextuples. Also, given a ring of four circles, formed by two pairs of circles in 


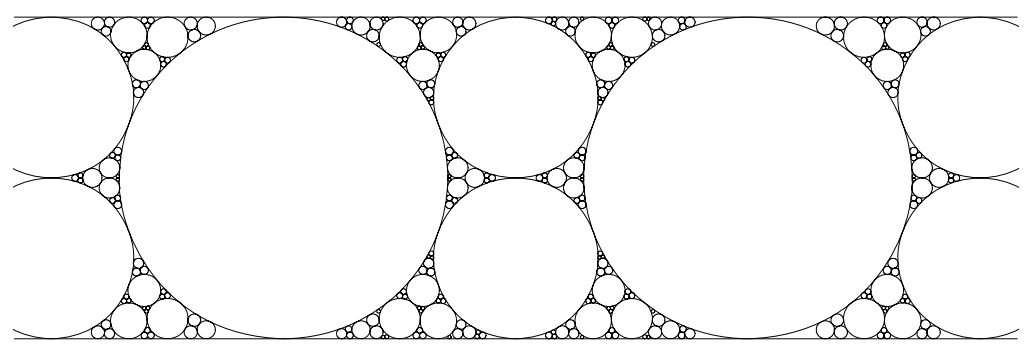

Figure 3: Another generalized Apollonian packing.

a sextuple, there is a quadratic equation ((6) below) whose coefficients involve the bends of these four circles, the roots of which are the bends of the other pair of circles in the sextuple. These results generalize the classical Descartes equation ((2) below). Replacing each bend by the corresponding bend*(complex) center in each of these equations gives results which generalize the "Complex Descartes Theorem" of [8].

(ii) There is an analog of "Descartes reflection" (see [5]) in which three circles (one from each pair in a sextuple, these three circles occupying a curvilinear triangle formed by the other three circles of the sextuple) are replaced by three circles occupying the other triangle formed by these three circles, thus forming another sextuple. Given a sextuple, this operation can be performed in eight different ways. See Section 4. Iteration of this operation creates a generalized Apollonian packing in which the interiors of all circles are disjoint. A packing is determined by any three touching circles within it.

(iii) All six bends of the circles in a sextuple can be integers. See Section 5 . The integrality property is inherited by all derived circles.

(iv) if all bends of a sextuple are integers, the sextuple can be placed in the plane so that for each circle with bend $b$ and center $(x, y)$, the "bend times center" quantities $(b x, b y)$ are of the form $(m \sqrt{2}, n)$ with $m, n$ integers. This property is inherited by the generalized packing based on this sextuple. See Section 7.

(v) The construction of the generalized packing can be realised by integral linear operations acting on matrices representing the sextuples. These matrices could be $6 \times 4$ matrices with each row containing the "abbc" or "augmented bend, bend times center" coordinates introduced in [8], and defined in Section 2 below. However it is convenient to represent a sextuple by a $4 \times 4$ matrix in which the first three rows contain the abbc coordinates of three of the circles in the sextuple (one from each pair) and the fourth row 
is the average of the two rows that represent a pair of circles in the sextuple (this average is the same for each of the three pairs, see Lemma 4 below). This row does not represent a circle. See Section 3.

(vi) There are dual operations acting on the right, which represent Moebius transformations. See Section 3.

(vii) Among the sextuples with integer bends, there are "root" sextuples (see [4] and [6]) having the property that any application of the reflection operation in (ii) results in circles with larger bends. These root sextuples can be found by applying a reduction algorithm, just as in the Descartes case. Except for the special sextuple with bends $(0,2 ; 0,2 ; 1,1)$ (Figure $1(\mathrm{a})$ ), each root sextuple has exactly one circle with negative bend. We have a conjecture (7 below) as to the number of primitive root sextuples with smallest bend $-n$. See Section 6.

(viii) By inverting a generalized Apollonian packing in each circle in the packing, and then again in every circle, and so on, we obtain a "generalized Apollonian super-packing", directly analogous to the Apollonian super-packing studied in [6]. There is essentially just one super-packing in which all bends are integral. This super-packing can be placed in the plane, in exactly four ways, so that each $b x / \sqrt{2}, b y$ is integral. In each version of this super-packing, there is a basic rectangle $(0, \sqrt{2}) \times(0,1)$ which repeats by translation and reflection to cover the whole plane. See Section 7 and Figure 4.

(ix) Each primitive integral sextuple appears exactly once in the $(0, \sqrt{2}) \times$ $(0,1)$ rectangle of the super-packing. See Section 7 . Computation suggests that there are some symmetries within the basic rectangle, like those shown in [6]. See Figures 5(a)-(d) below.

(x) One can consider "ball-bearing" structures of circles, in which a ring of $n$ balls (each touching two neighbors) have the property that there exist "inner" and "outer" circles that each touch each of the "balls" in the ring. The case $n=3$ reproduces the Descartes configuration; the case $n=4$ gives the sextuples studied in this paper. The bends of all $n+2$ circles can be integral only when $n=3,4,6$. There is a quadratic equation (equation (8) below) whose roots are the bends of the "inner" and "outer" circles, and whose coefficients involve the bends of the circles in the ring. See Section 8.

(xi) There is an analog of the Farey series and the associated Ford circles, in which at every stage we insert two new fractions (and two new touching circles) instead of just one, between every existing adjacent pair of fractions. See Section 9 and Figure 6 below. 


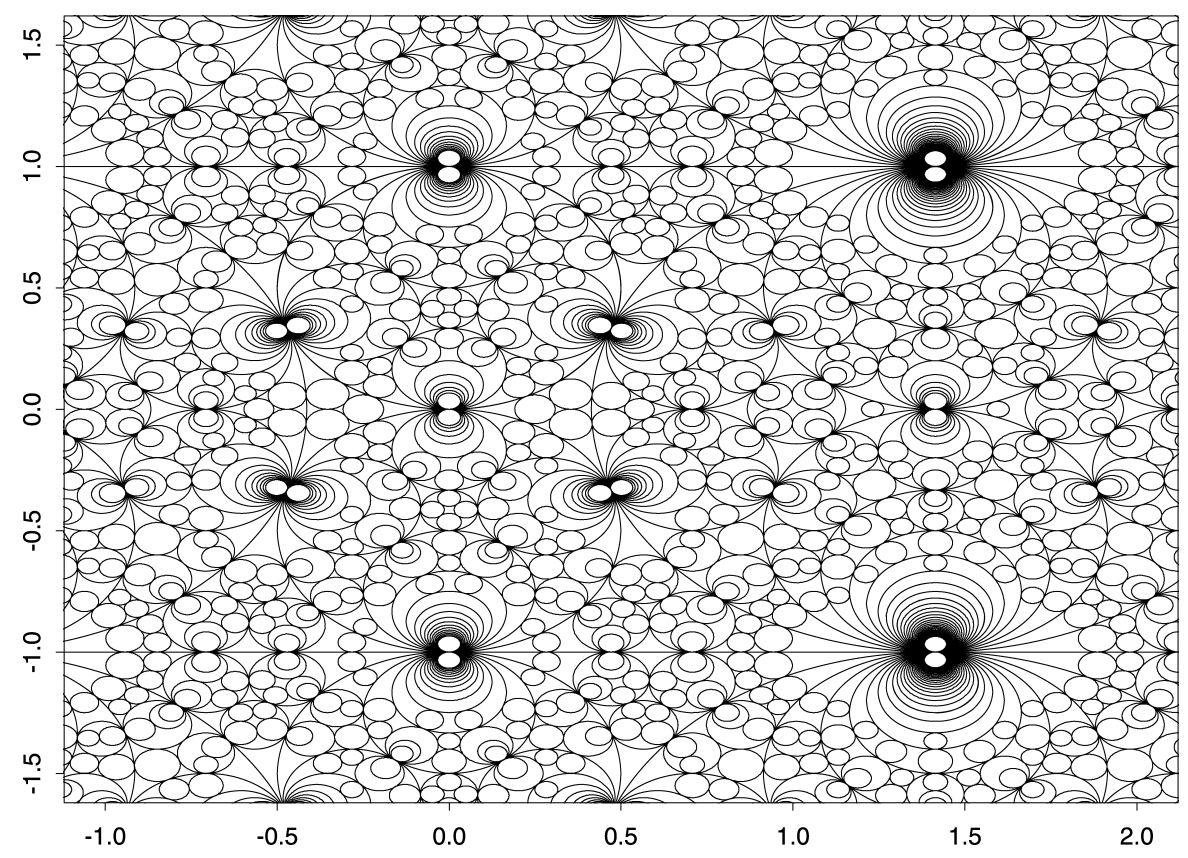

Figure 4: A generalized Apollonian super-packing.

\section{Conventions and notation}

We adopt the conventions in [4-8]. A circle $C$ with radius $r$ and center $(x, y)$ is described by its "augmented bend, bend*center" (abbc) coordinates $\mathbf{a}(C)=(\bar{b}, b, b x, b y)$ where $b$ is the bend (i.e. curvature) $b=1 / r$, and where $\bar{b}$ is the bend of the circle that is the inverse of $C$ in the unit circle, namely

$$
\bar{b}=b\left(x^{2}+y^{2}\right)-1 / b
$$

Sometimes we will write $z=x+i y$ and work in the complex plane.

A straight line is a circle with bend zero, and we need a definition to replace (1). Consider the line with equation $p_{1} x+p_{2} y=h$ where $p=\left(p_{1}, p_{2}\right)$ is a unit vector. This is the limit as $\lambda \rightarrow \infty$ of a circle with center $\lambda p$ and radius $(\lambda-h)$, so it makes sense to define the abbc coordinates of this line as $\left(2 h, 0, p_{1}, p_{2}\right)$. If the bend of a circle is positive, the "interior" of the circle contains its center. In all cases, the abbc coordinates uniquely determine the circle and its orientation. We say that two circles "touch" or "are tangent" when they have just one point in common and their interiors are disjoint. 
We are considering sets of six circles such that each touches four others, and the interiors are all disjoint.

We can describe a sextuple $S$ by the $6 \times 4$ matrix that has the abbc coordinates of the circles in its rows, where the circles are taken in what we call standard order, namely rows 1,2 contain the circles in a pair, as do rows 3,4 and rows 5,6. Since we can permute the three pairs, and also can take the circles of a pair in either order, there are 48 different standard orders, all representing the same sextuple.

We introduce the following matrix $W$. This is twice the inverse of what is called the "Wilker quadratic form" in [5], so named in honor of Wilker [13].

$$
\mathbf{W}=\left(\begin{array}{rrrr}
0 & -1 / 2 & 0 & 0 \\
-1 / 2 & 0 & 0 & 0 \\
0 & 0 & 1 & 0 \\
0 & 0 & 0 & 1
\end{array}\right)
$$

From [5] and [8] we have

Lemma 1. For any two circles $C$ and $C^{\prime}$,

$$
\begin{aligned}
\mathbf{a}(C) \mathbf{W a}\left(C^{\prime}\right)^{T} & =1 & & \text { if } C=C^{\prime} \\
& =-1 & & \text { if } C \text { and } C^{\prime} \text { are externally tangent }
\end{aligned}
$$

Proof. Simple algebra.

We also introduce the matrix of the "Descartes quadratic form" (twice what is defined in [5])

$$
\mathbf{D}=\left(\begin{array}{rrrr}
1 & -1 & -1 & -1 \\
-1 & 1 & -1 & -1 \\
-1 & -1 & 1 & -1 \\
-1 & -1 & -1 & 1
\end{array}\right)
$$

Note that $\mathbf{D}^{-1}=\mathbf{D} / 4$. We honor Rene Descartes here because in 1643 he derived a result connecting the bends of four mutually tangent circles, equivalent to

$$
2\left(b_{1}^{2}+b_{2}^{2}+b_{3}^{2}+b_{4}^{2}\right)=\left(b_{1}+b_{2}+b_{3}+b_{4}\right)^{2}
$$

and this can be written $\mathbf{b} \mathbf{D} \mathbf{b}^{T}=0$ where $\mathbf{b}$ is the vector $\left(b_{1}, b_{2}, b_{3}, b_{4}\right)$. Given a Descartes configuration consisting of four mutually tangent circles 
$C_{1}, C_{2}, C_{3}, C_{4}$ with disjoint interiors, we define the $4 \times 4$ matrix $\mathbf{A}$ as containing rows $\mathbf{a}\left(C_{j}\right), j=1,2,3,4$. From Lemma 1 we have

$$
\mathbf{A W A}^{T}=\mathbf{D}
$$

Inverting both sides of this relation we immediately obtain the "Augmented Euclidean Descartes Theorem" of [8], namely

$$
\mathbf{A}^{T} \mathbf{D A}=4 \mathbf{W}^{-1}
$$

The Descartes equation (2) is the second diagonal element of this relation. From the $(3,4)$ coordinates we have the "Complex Descartes equation" of [8], namely

$$
(\mathbf{b} * \mathbf{z}) \mathbf{D}(\mathbf{b} * \mathbf{z})^{T}=0
$$

where $\mathbf{b} * \mathbf{z}=\left(b_{1} z_{1}, b_{2} z_{2}, b_{3} z_{3}, b_{4} z_{4}\right)$. We will find generalizations of this and (2) in the sextuple case, but the same argument will not work because we have a $6 \times 4$ matrix of abbc coordinates, and we get singular matrices.

The following Lemma will be very useful.

Lemma 2 ([5], Appendix A). The effect of an arbitrary Moebius transformation on the abbc vector $\mathbf{a}(C)$ describing a circle $C$ is to replace $\mathbf{a}(C)$ by $\mathbf{a}(C) \mathbf{m}$ where $\mathbf{m}$ is an element of a certain group Moeb of $4 \times 4$ matrices. All matrices in this group satisfy $\mathbf{m} \mathbf{W} \mathbf{m}^{T}=\mathbf{W}$.

The proof is straightforward, using the following description of the elements of Moeb.

The group Moeb is generated by matrices of the following kinds:

scaling by $\lambda: \mathbf{m}$ is diagonal, with elements $(1 / \lambda, \lambda, 1,1)$.

rigid rotation about the origin: $\mathbf{m}$ is the $4 \times 4$ unit matrix, with the $(3,4) \times(3,4)$ submatrix replaced by

$$
\left(\begin{array}{rr}
\cos \theta & \sin \theta \\
-\sin \theta & \cos \theta
\end{array}\right) \text {. }
$$

inversion in the unit circle: $\mathbf{m}$ replaces the leading $2 \times 2$ submatrix of the $4 \times 4$ unit matrix by

$$
\left(\begin{array}{ll}
0 & 1 \\
1 & 0
\end{array}\right) .
$$


translation by $x, y$ :

$$
\mathbf{m}=\left(\begin{array}{cccc}
1 & 0 & 0 & 0 \\
x^{2}+y^{2} & 1 & x & y \\
2 x & 0 & 1 & 0 \\
2 y & 0 & 0 & 1
\end{array}\right)
$$

\section{Sextuples}

Lemma 3. If $C$ and $C^{\prime}$ are a pair of (non-tangent) circles in a sextuple configuration, then

$$
\mathbf{a}(C) \mathbf{W a}\left(C^{\prime}\right)^{T}=-3 .
$$

Proof. The relation is easily verified for the special sextuple shown in Figure 1 (c), with the origin at the contact-point of the two circles with bend 2 , for which the abbc coordinates are the rows of

$$
\left(\begin{array}{rrrr}
2 & 0 & 0 & 1 \\
4 & 2 & 0 & -1 \\
2 & 0 & 0 & -1 \\
4 & 2 & 0 & 1 \\
1 & 1 & \sqrt{2} & 0 \\
1 & 1 & -\sqrt{2} & 0
\end{array}\right)
$$

Any sextuple can be obtained from this one by a Moebius transformation. By Lemma 2, the effect of a Moebius transformation is to replace $\mathbf{a}(C)$ and $\mathbf{a}\left(C^{\prime}\right)$ by $\mathbf{a}(C) \mathbf{m}$ and $\mathbf{a}\left(C^{\prime}\right) \mathbf{m}$ respectively, for some $\mathbf{m}$ in Moeb. Each such $\mathbf{m}$ satisfies $\mathbf{m} \mathbf{W} \mathbf{m}^{T}=\mathbf{W}$ and the Lemma follows.

This simple result has an important consequence.

Lemma 4. In a sextuple $S$ containing circles $C_{1}, C_{1}^{\prime}$ etc. we have

$$
\mathbf{a}\left(C_{1}\right)+\mathbf{a}\left(C_{1}^{\prime}\right)=\mathbf{a}\left(C_{2}\right)+\mathbf{a}\left(C_{2}^{\prime}\right)=\mathbf{a}\left(C_{3}\right)+\mathbf{a}\left(C_{3}^{\prime}\right) .
$$

Proof. For $j=1,2,3$ define $\mathbf{w}_{j}=\left(\mathbf{a}\left(C_{j}\right)+\mathbf{a}\left(C_{j}^{\prime}\right)\right) / 2$, and let $\mathbf{F}_{j}$ be the $4 \times 4$ matrix whose rows are $\mathbf{a}\left(C_{1}\right), \mathbf{a}\left(C_{2}\right), \mathbf{a}\left(C_{3}\right), \mathbf{w}_{j}$. From Lemmas 1 and 3 we have that $\mathbf{w}_{1} \mathbf{W} \mathbf{w}_{1}^{T}=(1-3-3+1) / 4=-1$, and $\mathbf{w}_{1} \mathbf{W} \mathbf{w}_{2}^{T}=(-1-1-1-$ 1) $/ 4=-1$ also. So

$$
\mathbf{F}_{1} \mathbf{W} \mathbf{F}_{1}^{T}=\mathbf{K}=\mathbf{F}_{2} \mathbf{W F}_{1}^{T}
$$


where

$$
\mathbf{K}=\left(\begin{array}{rrrr}
1 & -1 & -1 & -1 \\
-1 & 1 & -1 & -1 \\
-1 & -1 & 1 & -1 \\
-1 & -1 & -1 & -1
\end{array}\right)
$$

Since all the matrices in (4) are non-singular, this shows that $\mathbf{F}_{1}=\mathbf{F}_{2}$, so that $\mathbf{w}_{1}=\mathbf{w}_{2}$. Similarly both are equal to $\mathbf{w}_{3}$.

This result makes possible a convenient representation of a sextuple.

F notation. Given a sextuple $S$ containing six circles $C_{1}, C_{1}^{\prime} ; C_{2}, C_{2}^{\prime} ; C_{3}, C_{3}^{\prime}$, an $F$ matrix describing the sextuple is a $4 \times 4$ matrix whose first three rows contain the abbc coordinates of three of the circles (one from each pair) and the fourth row is the average of the abbc coordinates of the two circles in any pair.

Given a sextuple, there are 48 such representations, obtained by choosing the pairs in different orders, and choosing different representatives of each pair. We can move between different representations by premultiplying $\mathbf{F}$ by matrices like

$$
\left(\begin{array}{rrrr}
0 & -1 & 0 & 2 \\
0 & 0 & -1 & 2 \\
1 & 0 & 0 & 0 \\
0 & 0 & 0 & 1
\end{array}\right)
$$

which replaces $C_{1}, C_{2}, C_{3}$ by $C_{2}^{\prime}, C_{3}^{\prime}, C_{1}$. The set of 48 such integral matrices form a group which we call Perm.

We can recover all the $486 \times 4$ standard representations as $\mathbf{G}(S)=$ $\mathbf{E F}(S)$ where

$$
\mathbf{E}=\left(\begin{array}{rrrr}
1 & 0 & 0 & 0 \\
-1 & 0 & 0 & 2 \\
0 & 1 & 0 & 0 \\
0 & -1 & 0 & 2 \\
0 & 0 & 1 & 0 \\
0 & 0 & -1 & 2
\end{array}\right)
$$

Lemma 2 above pointed out that the effect of a Moebius transformation on the abbc coordinates of a circle is right-multiplication by an element $\mathbf{m}$ of a certain group. Since such a transformation retains adjacency properties, it follows that the effect on an $\mathbf{F}$ matrix describing a sextuple is to replace $\mathbf{F}$ by $\mathbf{F m}$.

The next Theorem generalizes the Descartes equation, in two ways. 
Theorem 1. Given three mutually tangent circles with bends $b_{1}, b_{2}, b_{3}$, enclosing two triangular gaps, the bends of the two sets of three circles that can be inscribed in these gaps are $\left(2 w-b_{1}, 2 w-b_{2}, 2 w-b_{3}\right)$ and $\left(2 w^{\prime}-b_{1}, 2 w^{\prime}-\right.$ $\left.b_{2}, 2 w^{\prime}-b_{3}\right)$ where $w, w^{\prime}$ are the roots of the equation

$$
w^{2}-2 w\left(b_{1}+b_{2}+b_{3}\right)+b_{1}^{2}+b_{2}^{2}+b_{3}^{2}=0
$$

A similar result holds for the bend*centers. Also, given the bends $b_{1}, b_{1}^{\prime}, b_{2}, b_{2}^{\prime}$ of the circles in two pairs of a sextuple, forming a ring, the bends of the other pair, each of which touches all four of these circles, are the roots of the equation

$$
2 x^{2}-x \sigma+\tau-\frac{3}{8} \sigma^{2}=0
$$

where $\sigma=b_{1}+b_{1}^{\prime}+b_{2}+b_{2}^{\prime}, \tau=b_{1}^{2}+b_{1}^{\prime 2}+b_{2}^{2}+b_{2}^{\prime 2}$. The same equation holds when each bend $b$ is replaced by bz, where $z$ is the (complex) center of the circle with bend $b$.

Proof. If $w$ is the $(4,2)$ element of the $\mathrm{F}$ matrix that describes a sextuple, so that $2 w=b_{1}+b_{1}^{\prime}=b_{2}+b_{2}^{\prime}=b_{3}+b_{3}^{\prime}$, then inverting both sides of the equation $\mathbf{F W} \mathbf{F}^{T}=\mathbf{K}$ we find

$$
\mathbf{F}^{T} \mathbf{K}^{-1} \mathbf{F}=\mathbf{W}^{-1}
$$

in which

$$
\mathbf{K}^{-1}=\frac{1}{2}\left(\begin{array}{rrrr}
1 & 0 & 0 & -1 \\
0 & 1 & 0 & -1 \\
0 & 0 & 1 & -1 \\
-1 & -1 & -1 & 1
\end{array}\right) .
$$

The $(2,2)$ element of this equation shows that (5) holds. Also, since $b_{1}^{\prime}=2 w-b_{1}, b_{2}^{\prime}=2 w-b_{2}$, we have

$$
\sigma=4 w, \quad \tau=2\left(b_{1}^{2}+b_{2}^{2}\right)-4 w\left(b_{1}+b_{2}\right)+8 w^{2}
$$

and (6) follows immediately. The bend*center results follow by the same argument.

This validates claim (i) of the Introduction. Equations (5) and (6) can each be regarded as generalizing the Descartes equation. 


\section{Reflection and generalized Apollonian packing}

In [5] the operation of "Descartes reflection" was defined, in which one of the circles in a Descartes quadruple is replaced by the other circle that is tangent to the same three circles as the chosen one. Given three mutually tangent circles $C_{1}, C_{2}, C_{3}$ with bends $b_{1}, b_{2}, b_{3}$ and (complex) centers $z_{1}, z_{2}, z_{3}$, the bends $b_{4}, b_{4}^{\prime}$ of the two circles that are each tangent to these three circles are the roots of the Descartes equation (2). So "reflection" of the $b_{4}$ circle replaces this circle by one with bend $b_{4}^{\prime}=2\left(b_{1}+b_{2}+b_{3}\right)-b_{4}$, and (using the "Generalized Descartes equation") with bend*center $b_{4}^{\prime} z_{4}^{\prime}=2\left(b_{1} z_{1}+b_{2} z_{2}+\right.$ $\left.b_{3} z_{3}\right)-b_{4} z_{4}$.

This operation can be performed in four ways, taking each of the circles in the Descartes quadruple in turn.

Using equation (5), we can perform a similar "reflection" operation on the circles in a sextuple. Specifically, let $\mathbf{F}$ be a $4 \times 4$ matrix describing a sextuple. Then (5) has two roots $w, w^{\prime}$ say. One of these gives the bends of the other three circles in the sextuple described by $\mathbf{F}$, namely $2 w-b_{1}, 2 w-$ $b_{2}, 2 w-b_{3}$. The other root gives the bends of another set of three circles, namely $2 w^{\prime}-b_{1}, 2 w^{\prime}-b_{2}, 2 w^{\prime}-b_{3}$ which also form a sextuple with the three circles in the first three rows of $\mathbf{F}$. The (complex) centers of these circles can be found by using (5) with the bends replaced by bend*centers. The new sextuple is the result of inverting the original one in the circle that passes through the three points of contact of the three circles that stay invariant. This validates claim (ii).

Since from (5) $w+w^{\prime}=2\left(b_{1}+b_{2}+b_{3}\right)$, we do not need to solve a quadratic explicitly.

Lemma 5. The operation of reflection in circles $C_{1}, C_{2}, C_{3}$ can be performed by premultiplying an $\mathbf{F}$ matrix that describes the sextuple, with the abbc coordinates of these three circles in its first three rows, by the matrix $\mathbf{R}$, where

$$
\mathbf{R}=\left(\begin{array}{rrrr}
1 & 0 & 0 & 0 \\
0 & 1 & 0 & 0 \\
0 & 0 & 1 & 0 \\
2 & 2 & 2 & -1
\end{array}\right)
$$

Proof. $R$ replaces $w$ by $w^{\prime}$ and $\mathbf{w}$ by $\mathbf{w}^{\prime}$.

This validates the "reflect" part of claim (v). Note that reflection is an involution: $\mathbf{R}^{2}=\mathbf{I}$. $\mathbf{R}$ is the same as the matrix $S_{4}$ of [4] that implements reflection of the fourth circle in a Descartes quadruple. To reflect in a different triad of circles in the sextuple, we use the "permute" group Perm to 
bring the required triad of circles into rows $1,2,3$ of the representation $\mathbf{F}$. So the operation can be performed in eight ways. Note that each of these eight operations puts a new triad of circles into a different one of the gaps formed by the original six circles.

The generalized Apollonian packing based on a given sextuple $S_{0}$ is obtained by premultiplying $\mathbf{F}\left(S_{0}\right)$ by "permute" and "reflect" matrices, in all possible ways. Thus a general sextuple in the generalized Apollonian packing based on $S_{0}$ is represented by an $\mathbf{F}$ matrix of the form

$$
\mathbf{R P}_{n} \mathbf{R} \mathbf{P}_{n-1} \cdots \mathbf{R} \mathbf{P}_{1} \mathbf{F}\left(S_{0}\right)
$$

(Since $\mathbf{R}$ is an involution, we do not need powers of $\mathbf{R}$ higher than the first; since the "permute" matrices form a group, we never need more than one between two R's.)

Figures 2 and 3 show generalized Apollonian packings based on the sextuples in Figure 1(a) and 1(c) respectively. Note that in such packings, the interiors of all circles are disjoint (a circle with negative bend has the point at infinity in its interior) and no circles intersect (though many touch).

As in the Descartes case, there are several ways to regard the generalized Apollonian packing:

(i) as a packing of circles in the plane, each touching four others; these four all being touched by one other circle;

(ii) as a packing of triads of touching circles, each triad surrounding (and being surrounded by) another triad of circles;

(iii) as an arrangement formed by configurations consisting of sextuples of circles, interlocking by overlapping sets of three tangent circles.

The view (iii) gives rise to intriguing group-theory problems, analogous to those studied in Graham et al [4-5].

\section{Integral packings}

We will consider only primitive sextuples, where the bends have no common factor. Suppose $\left(a, a^{\prime} ; b, b^{\prime} ; c, c^{\prime}\right)$ is a primitive integral sextuple. Since $2 w=$ $a+a^{\prime}$ etc., $2 w$ is a positive integer. Since from (5)

$$
w=a+b+c \pm \sqrt{2(a b+a c+b c)}
$$

$a b+a c+b c$ must be twice a square, say $2 m^{2}$ with $m$ an integer, and $w$ is an integer. 
At least one of $a, b, c$ must be odd, since otherwise all six bends are even. Suppose $a$ is odd. Then

$$
(a+b)(a+c)=a^{2}+2 m^{2}
$$

which is odd, so each of $a+b$ and $a+c$ must be odd, and $b$ and $c$ must be even. Hence $w=a+b+c \pm 2 m$ is an odd positive integer.

We can generate (all) primitive integral sextuples of bends by choosing $a$ odd and $m$ arbitrarily (without loss of generality we can take $m$ nonnegative, but $a$ can have either sign). Factorize $a^{2}+2 m^{2}$ as $f g$, with $f$ and $g$ positive, and set $b=f-a, \quad c=g-a, \quad w=a+b+c \pm 2 m, \quad a^{\prime}=2 w-a$ etc. The sextuple is primitive iff $a, f, g$ have no common factor.

This settles claim (iii).

Lemma 6. If the bends of a sextuple are all integers, the same is true for the bends of all the circles in the generalized packing based on this sextuple. If the abbc coordinates of the circles in a sextuple are of the form (integer, integer, integer $\sqrt{2}$, integer), then the same is true for every circle in the packing.

Proof. The matrix $\mathbf{R}$ and the matrices in the group Perm have integer entries.

This lemma is not empty, since we have the examples in figure 1(a) and $1(\mathrm{c})$.

\section{Reduction algorithm and root sextuples}

The size of a sextuple is conveniently measured by the value of $w$. Given the bends of a sextuple, by applying a permutation and then reflecting, we may be able to find a sextuple with smaller value of $w$. If this is not possible, we call this a root sextuple. Except for the special sextuple (shown in Figure $1(\mathrm{c}))$ with bends $(0,2 ; 0,2 ; 1,1)$, a root sextuple has exactly one negative bend. As in the Descartes case (see [4]), starting from a given sextuple, there is a root sextuple that can be reached by repeated reflections. The root sextuple is unique because its three smallest bends are the bends of the three largest circles in the packing, and so are unique. However the packing may contain more than one copy of this sextuple, for example the packing in Figure 2 contains two copies of the root sextuple $(-1,7 ; 2,4 ; 2,4)$ and the packing in Figure 3 contains infinitely many copies of the root sextuple $(0,2 ; 0,2 ; 1,1)$. 
Lemma 7. The sextuple described by integer bends $a \leq b \leq c \leq w$ (satisfying (5)) is a root sextuple iff $w \leq a+b+c$.

Proof. $w^{\prime} \geq w$, and this is true also if we replace any of $a, b, c$ by $a^{\prime}, b^{\prime}, c^{\prime}$.

Computation finds the numbers of primitive root sextuples with smallest bend $-n$, for $n=1, \ldots, 100$, in the following table.

The number of root sextuples with smallest bend $-n$.

$\begin{array}{lrrrrrrrrrr}i & 1 & 2 & 3 & 4 & 5 & 6 & 7 & 8 & 9 & 10 \\ n=0+i & 1 & 2 & 1 & 3 & 2 & 4 & 3 & 5 & 2 & 8 \\ n=10+i & 3 & 6 & 4 & 10 & 4 & 9 & 5 & 8 & 5 & 14 \\ n=20+i & 5 & 12 & 7 & 10 & 8 & 16 & 5 & 18 & 8 & 16 \\ n=30+i & 9 & 17 & 6 & 18 & 13 & 14 & 10 & 20 & 8 & 26 \\ n=40+i & 11 & 20 & 11 & 22 & 10 & 26 & 13 & 18 & 15 & 32 \\ n=50+i & 9 & 30 & 14 & 20 & 16 & 34 & 10 & 32 & 15 & 28 \\ n=60+i & 16 & 34 & 13 & 33 & 22 & 24 & 17 & 34 & 13 & 52 \\ n=70+i & 19 & 26 & 19 & 40 & 16 & 38 & 21 & 32 & 21 & 50 \\ n=80+i & 14 & 42 & 21 & 36 & 25 & 44 & 16 & 42 & 23 & 40 \\ n=90+i & 29 & 50 & 17 & 50 & 28 & 34 & 25 & 58 & 16 & 62\end{array}$

For example, the three primitive root sextuples with smallest bend -4 are:

$(-4,26 ; 7,15 ; 10,12)$

$(-4,30 ; 6,20 ; 13,13)$

$(-4,46 ; 5,37 ; 20,22)$.

From the counts in the table and several more with larger values of $n$, we conjecture a formula (having some resemblance to the one proved in [4] for the corresponding Descartes case) for the number of root sextuples.

Conjecture. The number of primitive root sextuples with smallest bend $-n$ is

$$
k(n)=\frac{1}{4}\left(n \prod_{p \mid n} \frac{p+\chi(p)}{p}+r(n)\right)
$$


where $\chi(2)=2, \chi(p)=-1,-1,1,1$ for $p=1,3,5,7(\bmod 8)$, and the remainder term depends on the number of different odd prime factors of $n$, which we name $\rho(n)$ :

$$
\begin{aligned}
& \text { if } n \text { is odd, } \rho(n)=1 \text {, then } r(n)=\phi(p) \text { where } \phi(p)=4,2,2,4 \\
& \quad \text { when } p=1,3,5,7(\bmod 8) \text {. } \\
& \text { if } n \text { is odd and } \rho(n) \geq 2, r(n)=2^{\rho(n)} \\
& \text { if } n \text { is even, } r(n)=4.2^{\rho(n)} \text {. }
\end{aligned}
$$

This conjecture constitutes claim (vi).

\section{Inversion and the generalized super-packing}

Given a generalized Apollonian packing, we can invert the whole packing in every circle in the packing, and then again in every circle in the resulting packing, and so on. The result is what we call a "generalized super-packing" (compare [5]). In this packing, every two circles either touch, or are disjoint. See Figure 4.

It is convenient to regard the super-packing as being composed of sextuples. We can generate the sextuples of the super-packing by adding another generator to the generalized Apollonian group, representing inversion in the first circle of a general sextuple.

Lemma 8. Given a sextuple $S$ described by an $F$ matrix $\mathbf{F}(S)$, the result of inversion in the circle described by the top row of $\mathbf{F}$ is a sextuple $S^{\prime}$ with

$$
\mathbf{F}\left(S^{\prime}\right)=\mathbf{V F}(S)
$$

where

$$
\mathbf{V}=\left(\begin{array}{rrrr}
-1 & 0 & 0 & 0 \\
2 & 1 & 0 & 0 \\
2 & 0 & 1 & 0 \\
2 & 0 & 0 & 1
\end{array}\right)
$$

Proof. Suppose the rows of $F(S)$ are $\mathbf{a}, \mathbf{b}, \mathbf{c}, \mathbf{w}$. From (3.26) of [5] (or by simple geometry) we have that since each of the $\mathbf{b}, \mathbf{c}$ circles touches the $\mathbf{a}$ circle, their transformed abbc coordinates are given correctly. Since the $\mathbf{b}^{\prime}$ circle (whose abbc coordinates are $\mathbf{2 w}-\mathbf{b}$ ) also touches the a circle, its transformed abbc coordinates are $\mathbf{2 a}+\mathbf{2} \mathbf{w}-\mathbf{b}$ so that the new fourth row of $F$ is $(1 / 2)(\mathbf{2} \mathbf{a}+\mathbf{b}+\mathbf{2} \mathbf{a}+\mathbf{2} \mathbf{w}-\mathbf{b})=\mathbf{2} \mathbf{a}+\mathbf{w}$. Note that $\mathbf{V}$ is an involution. 
This validates claim (v).

The situation is exactly analogous to that of the standard Apollonian super-packing, see [6]. It is convenient to work with the matrix group $B$ that has eight generators of the form $\mathbf{P R P}^{T}$, with $\mathbf{P} \epsilon$ Perm, which reflect in the eight different triads in a sextuple, and six generators of the form $\mathbf{P V P}^{T}$ which each invert with respect to one of the circles in a sextuple. Explicitly, given a sextuple with circles $C_{1}, C_{2}, C_{3}, C_{1}^{\prime}, C_{2}^{\prime}, C_{3}^{\prime}$ described by an $F$ matrix with the first three rows corresponding to the first three of these circles, we have $\mathbf{R}_{000}=\mathbf{R}$ as given above, which reflects leaving the circles $C_{1}, C_{2}, C_{3}$ invariant,

$$
\mathbf{R}_{100}=\left(\begin{array}{cccc}
-3 & 4 & 4 & 4 \\
0 & 1 & 0 & 0 \\
0 & 0 & 1 & 0 \\
-2 & 2 & 2 & 3
\end{array}\right)
$$

which reflects leaving $C_{1}^{\prime}, C_{2}, C_{3}$ invariant, and similarly $\mathbf{R}_{010}$ and $\mathbf{R}_{001}$,

$$
\mathbf{R}_{110}=\left(\begin{array}{cccc}
-3 & -4 & 4 & 12 \\
-4 & -3 & 4 & 12 \\
0 & 0 & 1 & 0 \\
-2 & -2 & 2 & 7
\end{array}\right)
$$

which reflects leaving $C_{1}^{\prime}, C_{2}^{\prime}, C_{3}$ invariant, and similarly $\mathbf{R}_{101}$ and $\mathbf{R}_{011}$, and finally

$$
\mathbf{R}_{111}=\left(\begin{array}{cccc}
-3 & -4 & -4 & 20 \\
-4 & -3 & -4 & 20 \\
-4 & -4 & -3 & 20 \\
-2 & -2 & -2 & 11
\end{array}\right)
$$

which reflects leaving $C_{1}^{\prime}, C_{2}^{\prime}, C_{3}^{\prime}$ invariant. Also we have $\mathbf{V}_{1}=\mathbf{V}$ as above, which inverts with respect to the circle $C_{1}$, and similarly $\mathbf{V}_{2}$ and $\mathbf{V}_{3}$, and

$$
\mathbf{V}_{1^{\prime}}=\left(\begin{array}{cccc}
-5 & 0 & 0 & 12 \\
-2 & 1 & 0 & 4 \\
-2 & 0 & 1 & 4 \\
-2 & 0 & 0 & 5
\end{array}\right)
$$

which inverts with respect to the circle $C_{1}^{\prime}$, and similarly $\mathbf{V}_{2^{\prime}}$ and $\mathbf{V}_{3^{\prime}}$.

We can write a word in the super-group $B$ as $\mathbf{U}=\mathbf{U}_{n} \mathbf{U}_{n-1} \cdots \mathbf{U}_{1}$, in which each $\mathbf{U}_{i}$ is one of these generators $\mathbf{R}_{x}$ and $\mathbf{V}_{y}$. Each such word in $B$ has a normal form, obtained by cancelling all squares, and moving each 
appearance of each inversion $\mathbf{V}_{y}$ as far to the right (i.e. earlier) as possible, so that $\mathbf{V}_{y}$ follows a reflection that does not leave the circle $C_{y}$ invariant. Then the sextuples in the super-packing are in 1-1 correspondence with normal-form words in the super-group. We need to show that there are no identities in this group other than those described above.

Theorem 2. The generators $\mathbf{R}_{000}$ etc. and $\mathbf{V}_{1}$ etc described above are involutions. Also each $\mathbf{V}_{j}$ commutes with the four $\mathbf{R} s$ that leave $C_{j}$ invariant. There are no other identities among these generators.

Proof. Direct computation verifies the involution and commutativity relations. The hard part is showing that there are no other relations. The following argument mimics the key part of the proof of Theorem 3.1 in [6]. We consider the effect of applying an element of the group (i.e. a word in normal form) to an arbitrary basic sextuple $S_{0}$. There are two cases. First, the word contains no inversions. In this case, a reflection $\mathbf{R}$ that leaves circles $C_{1}, C_{2}, C_{3}$ invariant puts three circles inside one of the curvilinear triangles that they form. We can get out of this triangle only by repeating this reflection, but this is not allowed in a normal-form word until some other reflection has intervened, and then not all of $C_{1}, C_{2}, C_{3}$ are still members of the sextuple.

Second, suppose an inversion $\mathbf{V}_{j}$ appears. This puts five circles inside $C_{j}$, and we can only get outside of this circle by another application of $\mathbf{V}_{j}$. But in a normal form word, $\mathbf{V}_{j}$ cannot reappear until either (i) another $\mathbf{V}$, or (ii) an $\mathbf{R}$ that does not leave $C_{j}$ invariant, has intervened. In either case $C_{j}$ is no longer a member of the sextuple. Thus we can never get back to the original sextuple.

Theorem 3. The integral super-packing can be placed in the plane so that all bend*centers are of the form $(m \sqrt{2}, n)$ with $m$ and $n$ integral, in exactly four ways.

Proof. The argument exactly parallels that in the Descartes case, see Theorems 6.2 and 6.3 of [6]. Given the bends of any primitive integral sextuple $S$, there is an unique normal-form word $\mathbf{U}$ in the generators of the super-Apollonian group that reduces it to the special integral sextuple $S_{0}=$ $(0,2 ; 0,2 ; 1,1)$. This sextuple can be placed in the plane making $b x / \sqrt{2}$ and by integers for every circle, by making the straight lines parallel to the $\mathrm{x}$ axis, and with the point of contact of the two bend-2 circles at any point $m \sqrt{2}, n$ with $m$ and $n$ integral. Application of inversions in the bend- 0 circles and reflections leaving these circles invariant then fills the whole plane, and forms just four different placements of the whole figure, namely with the origin at 
(i) the center of a bend-1 circle,

(ii) the point of contact of a bend-1 circle and one of the lines,

(iii) the contact-point of the two bend-2 circles,

(iv) the point of contact of a bend-2 circle and one of the lines.

We get only four placements, not eight as in the Descartes case, because here we cannot reflect in the line $y=x$ while keeping the values of $b x / \sqrt{2}$ and by integral. In each placement, the basic rectangle $(0, \sqrt{2}) \times(0,1)$ repeats by reflections to cover the whole plane.

Now applying the elements of the word $\mathbf{U}$ in reverse order to the $\mathrm{F}$ matrix describing this placed basic sextuple $S_{0}$, we find exactly one placing of the sextuple $S$ within each version of the basic rectangle.

This validates claims (vii) and (viii) of the Introduction.

Computation suggests that there are some symmetries within the basic rectangle, as in the Descartes case, namely

circles with even bends appear symmetrically about $x=\sqrt{2} / 2$ circles with bends $=0 \bmod 8$ appear symmetrically about $x=$ $\sqrt{2} / 4$

circles with bends $=2$ or $=6 \bmod 8$ appear symmetrically about $y=1 / 2$

a circle with bend $=4 \bmod 8$ and center $x, y$ has a mate at $\sqrt{2} / 2-x, 1-y$

See Figures 5(a)-(d). Each circle in these figures is the largest circle of a unique root sextuple. For example, the three root sextuples with smallest bend -4 , which we listed in section 6 above, lie within the three largest circles in Figure 5(c). We do not see any symmetries for circles with odd bends.

Similar symmetries in the Descartes case were conjectured in [6] and proved by S. Northshield [11].

\section{Ball-bearing configurations}

We define a "ball-bearing" structure as consisting of a ring of circles, each touching two neighbors, with the property that there exist "inner" and "outer" circles that each touch each of the circles in the ring.

Theorem 4. In an n-ball ball-bearing structure, where the bends of the "ball" circles are (in order) $b_{1}, b_{2}, \ldots, b_{n}$, the bends of the "inner" and "outer" 


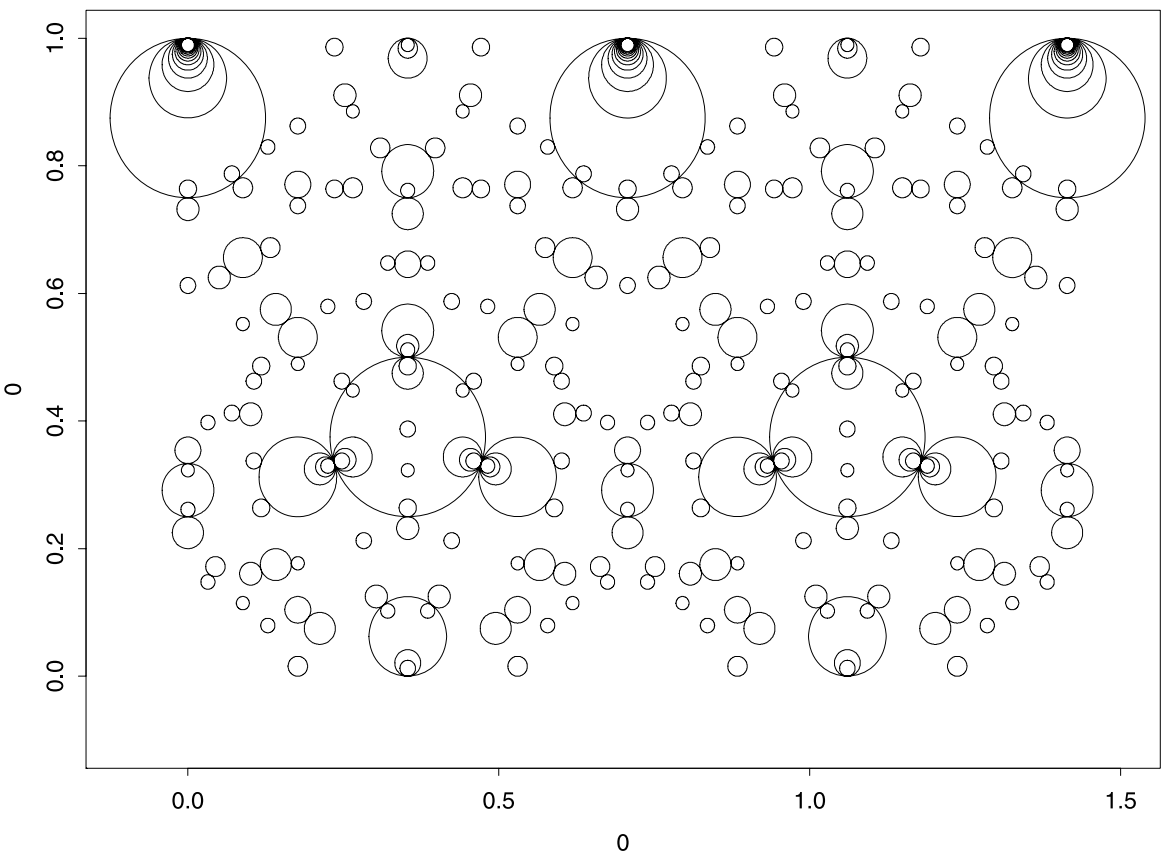

Figure 5: (a) Circles with bend $=0 \bmod 8$.

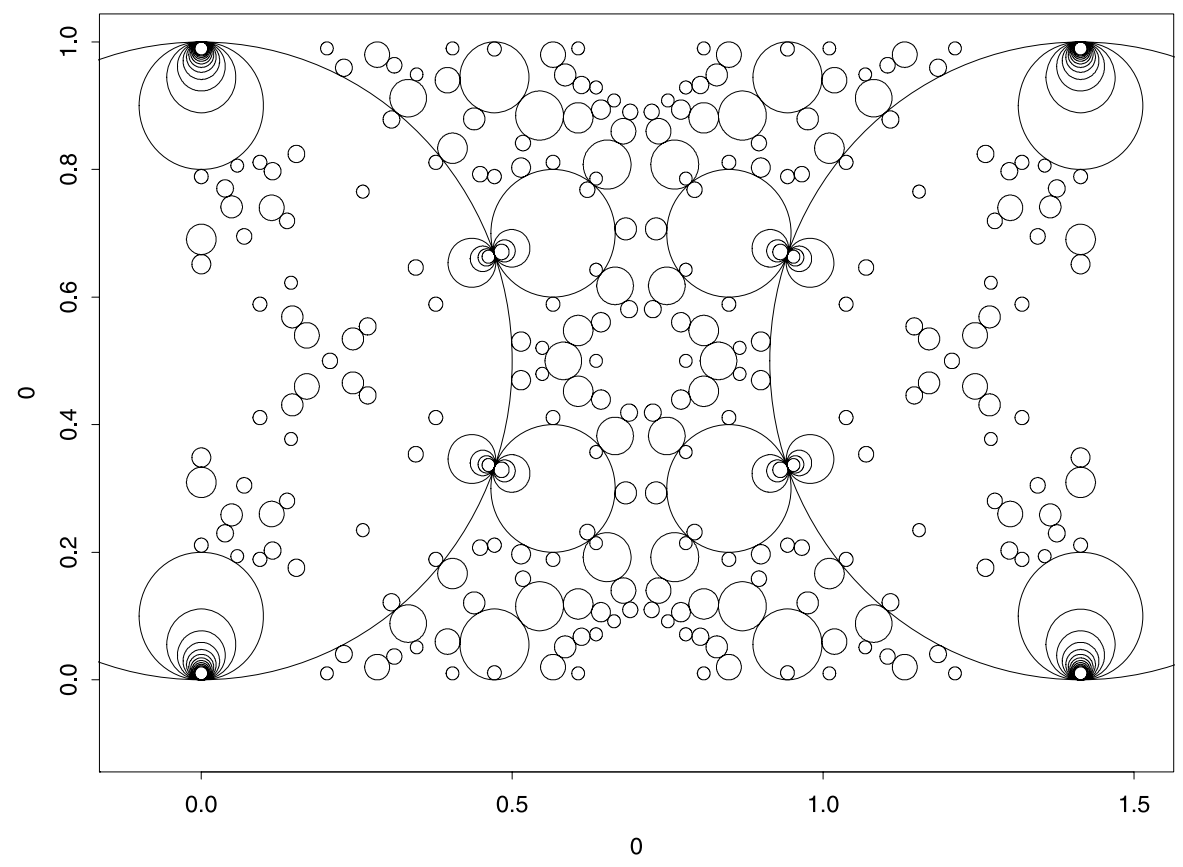

Figure 5: (b) Circles with bend $=2 \bmod 8$. 


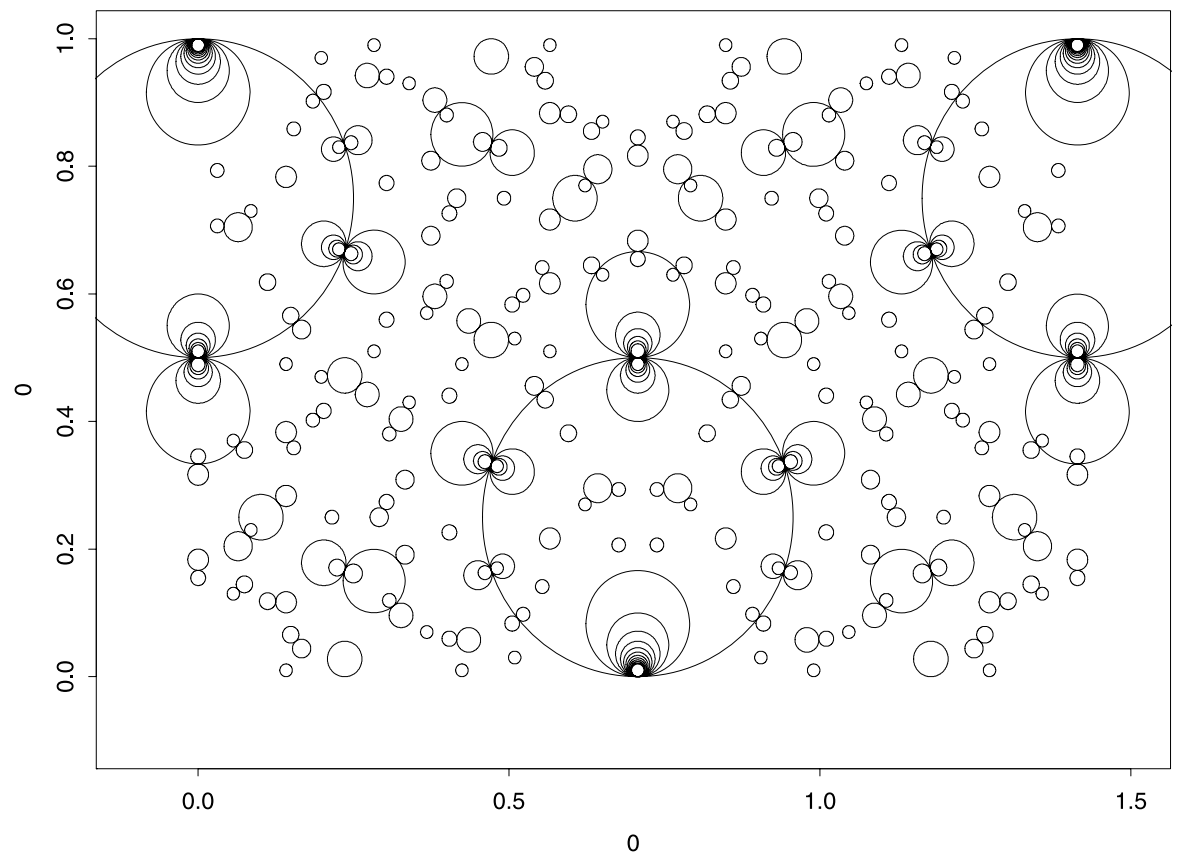

Figure 5: (c) Circles with bend $=4 \bmod 8$.

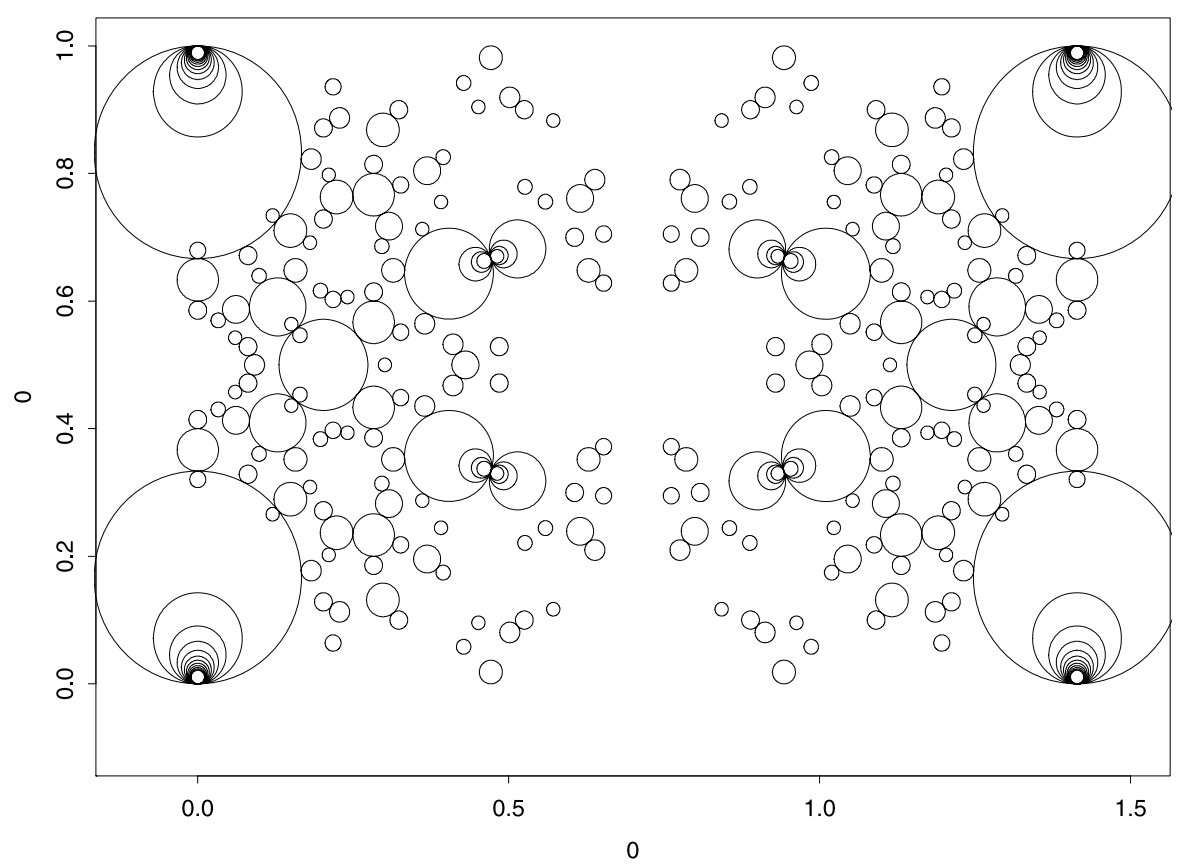

Figure 5: (d) Circles with bend $=6 \bmod 8$. 
circles are the roots of the equation

$$
D_{n} x^{2}-x \sigma+\tau-\frac{3}{2 n} \sigma^{2}=0
$$

where $\sigma=\sum b_{j}, \tau=\sum\left(b_{j}\right)^{2}$, and $D_{n}=(n / 2) \cot (\pi / n)^{2}$. Also, if the centers of the ring circles are the complex numbers $z_{1}, \ldots, z_{n}$, the bend ${ }^{*}$ center coordinates of the inner and outer circles are the roots of the equation

$$
D_{n} X^{2}-X S+T-\frac{3}{2 n} S^{2}=0
$$

where $S=\sum b_{j} z_{j}, T=\sum\left(b_{j} z_{j}\right)^{2}$.

Remark 1. For $n=3, D_{3}=1 / 2$ and (8) is the Descartes relation that connects the bends of four mutually tangent circles. Also (9) is then the "Complex Descartes Theorem" of [7].

Remark 2. An entry in MathWorld entitled "Soddy Circles" gives a formula that is different from ours. However this must be in error, since it is not homogeneous in the bends.

If the bends $b_{1}, b_{2}, \ldots, b_{n}$ are integers, the roots of (8) can be rational only when $n=3,4,6\left(D_{n}=1 / 2,2,9\right)$. Only in these cases can all the bends be integers.

Proof of the Theorem. We start by proving the relation (8) for a special configuration, in which the "inner" and "outer" circles are centered at the origin, with the $j$-th ring circle having center $\omega^{2 j}$, where $\omega^{n}=-1$. The radius of each ring circle is $s=\sin \pi / n$; the radii of the inner and outer circles are $1-s,-(1+s)$.

Let $a_{0}$ contain the abbc coordinates of the "inner" circle, and for $j=$ $1, \ldots, n$ let $a_{j}$ contain the abbc coordinates of the $j$-th circle in the ring, so that

$$
\begin{aligned}
& a_{0}=(s-1,1 /(1-s), 0,0) \\
& a_{j}=(1 / s-s, 1 / s, \cos (2 \pi j / n) / s, \sin (2 \pi j / n) / s) .
\end{aligned}
$$

We assemble these vectors in an $(n+1) \times 4$ matrix $\mathbf{A}$. Let $\mathbf{Q}$ be the $n+1 \times n+1$ matrix

$$
\mathbf{Q}=\left(\begin{array}{cc}
D_{n} & -\frac{1}{2} \mathbf{1}_{n}^{T} \\
-\frac{1}{2} \mathbf{1}_{n} & I_{n}-\frac{3}{2 n} \mathbf{1}_{n} \mathbf{1}_{n}^{T}
\end{array}\right)
$$

where $\mathbf{1}_{n}$ is the $n \times 1$ column vector $(1,1, \ldots, 1)^{T}$. The equation in the Theorem is $\mathbf{b}^{T} \mathbf{Q b}=0$ where $\mathbf{b}$ is the vector of bends $\mathbf{b}^{T}=\left(1 /(1-s),(1 / s) \mathbf{1}_{n}^{T}\right)$. 
Straightforward computation shows that

$$
\mathbf{A}^{T} \mathbf{Q A}=\frac{n}{s^{2}} \mathbf{W}^{-1}
$$

where $\mathbf{W}$ was defined in Section 2. Picking off the $(2,2)$ element proves the first claim of the theorem (for this special case). The second claim follows from the $(3,4) \times(3,4)$ submatrix.

Since for any $\mathbf{m}$ in $M o e b$ we have $\mathbf{m}^{T} \mathbf{W}^{-1} \mathbf{m}=\mathbf{W}^{-1}$, (8) remains true when $\mathbf{A}$ is replaced by $\mathbf{A m}$. Thus the relation holds for every ball-bearing configuration.

This validates claim (ix).

Remark 3. The bends of the ring circles satisfy $n-3$ linear identities, as do the "bend*center" quantities. For $n=4$ the identities are $b_{1}+b_{3}=b_{2}+b_{4}$ and $b_{1} z_{1}+b_{3} z_{3}=b_{2} z_{2}+b_{4} z_{4}$. For $n=6$ they are $b_{1}+b_{4}=b_{2}+b_{5}=b_{3}+b_{6}$, $b_{1}+b_{3}+b_{5}=b_{2}+b_{4}+b_{6}$, etc. For all $n \geq 3$ a ball-bearing configuration is determined by the abbc coordinates of the "inner" (or "outer") circle and any three consecutive "ring" circles. Thus we can work with $4 \times 4$ matrices in all cases.

Remark 4. Given three mutually tangent circles with integral bends $a, b, c$, set $q=a b+a c+b c$. Then the circles can belong to an integral Descartes quadruple iff $q$ is a perfect square; can belong to an integral sextuple (in the sense of this paper) iff $2 q$ is a perfect square; and can form part of a $n=6$ ball-bearing configuration iff $3 q$ is a perfect square.

Remark 5. In the $n=6$ case the basic integral configuration (analogous to the $(0,0,1,1)$ configuration in the $n=3$ case, and $(0,2 ; 0,2 ; 1,1)$ in the $n=4$ case) is $(1,1 ; 0,3,6,6,3,0)$, consisting of two parallel lines with two unit circles touching both, and a pile of four circles between them. This configuration can be placed in the plane so that all values of $b x / \sqrt{3}$ and $b y$ are integers. However it is not the case that all derived bends and bends*centers are integral.

Remark 6. One can also consider multiple-ring structures, for example a 1-5-5-1 structure. Here, every circle touches five others, and the circles have the symmetry of the vertices of an icosahedron. This suggests another generalization of Apollonian packing, in which nine circles are placed in every curvilinear triangle. In this case the bends cannot all be integers. 


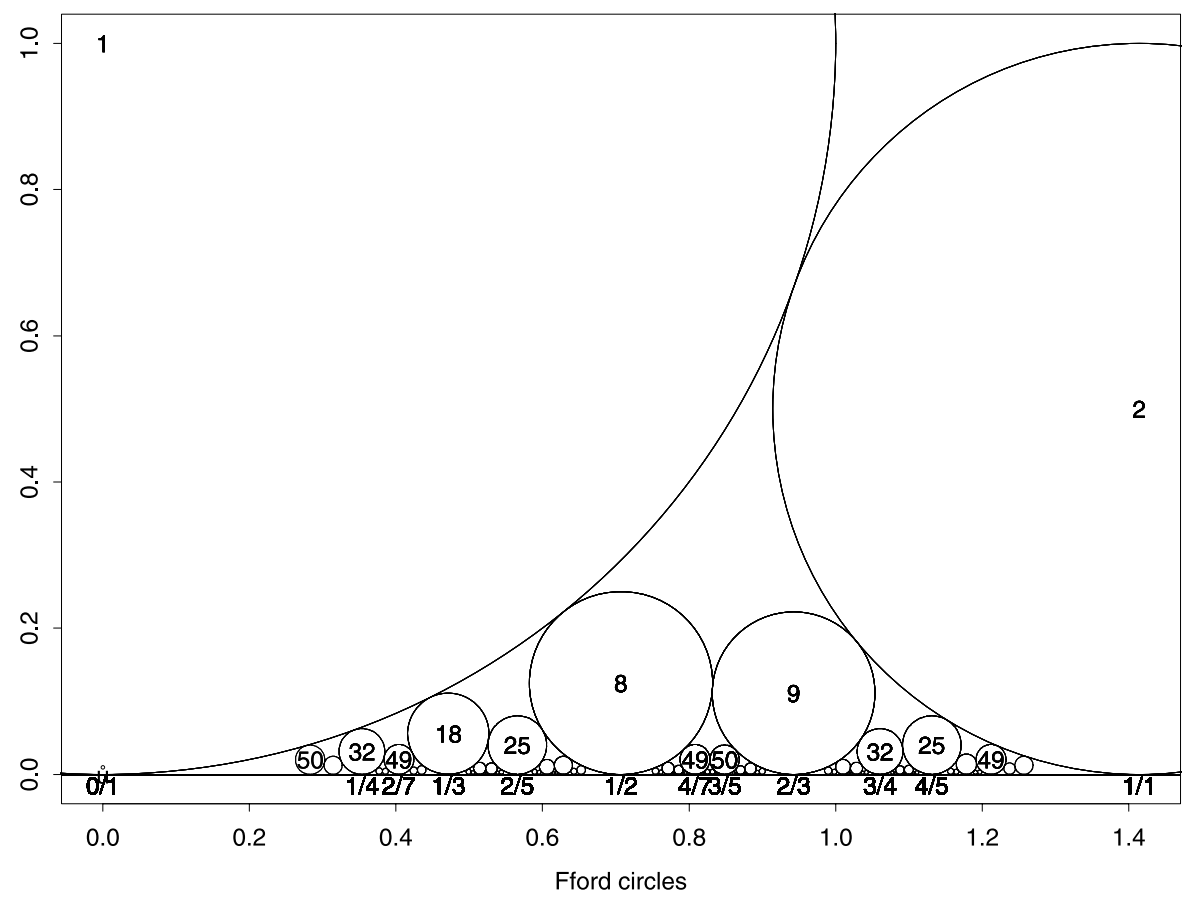

Figure 6: Generalized Ford circles.

Remark 7. Similar constructions are possible in three dimensions. Between four spheres, each of which touches the other three, we can (uniquely) pack four more spheres, touching each other and each touching three of the original spheres. We leave consideration of these configurations to another occasion.

\section{Generalized Farey series and Ford circles}

In the generalized Apollonian packing based on the sextuple $(0,2 ; 0,2 ; 1,1)$ depicted in Figure 3, the circles that touch the lower bend-zero line are analogous to Ford circles, and their points of contact are analogous to terms in the Farey series. See Figure 6 .

We start with a circle with bend 1 touching the $x$-axis at $x=0$, and another circle with bend 2, touching the first one and touching the $x$-axis at $x=\sqrt{2}$. Packing three circles between these (as in Figure $1(\mathrm{~d})$ ) gives two new circles touching the $x$-axis, namely a circle with bend 8 touching at $x=(1 / 2) \sqrt{2}$ and another with bend 9 touching at $x=(2 / 3) \sqrt{2}$. The 
third circle is ignored. Note that $8=2 \cdot 2^{2}, 9=3^{2}$, and $1 / 2=(0+1) /(1+1)$, $2 / 3=(0+2 \cdot 1) /(1+2 \cdot 1)$. This pattern continues. In general, in a gap between a circle with bend $q^{2}$ touching at $x=(p / q) \sqrt{2}$ and another with bend $2 s^{2}$ touching at $x=(r / s) \sqrt{2}$, where $p$ is even and $r$ is odd, we construct a circle with bend $(q+2 s)^{2}$ touching at $x=((p+2 r) /(q+2 s)) \sqrt{2}$ and another with bend $2(q+s)^{2}$ touching at $x=((p+r) /(q+s)) \sqrt{2}$. Two fractions $p / q, r / s$ are neighbors, and the corresponding circles touch each other, if and only if $|p s-q r|=1$. At every stage, the two types of circles (with bends $q^{2}$ and $\left.2 s^{2}\right)$ alternate. See Figure 6.

\section{Conclusion, and some open questions}

We have shown that much of the theory of the classical Apollonian packing and super-packing can be carried over to the sextuple case. We have left open several possible extensions of the theory. For example, what if we mix classical Apollonian insertions (one circle per gap) with the present rule of three circles per gap? Can all the bends be integral? We think not, except in cases where the initial triad of circles has some symmetry. What about placing six or ten circles in each gap? It seems that in all such cases we can use $4 \times 4$ matrices to describe the configurations that arise. We have not explored in depth the group theory associated with our generalized packings and super-packings.

Here are a few more questions.

Is the conjectured formula for the number of root sextuples correct?

Are the conjectured symmetries within the basic cell of the superpacking valid?

Do all integers arise as bends of circles in generalized Apollonian packings? (It is known that a positive fraction of possible integer bends does occur in a standard Apollonian packing, see [2]. It may be that all sufficiently large integers appear in each of our generalized packings.)

Is the Hausdorff dimension of our generalized packing the same as in the Apollonian case?

\section{Acknowledgment}

Thanks to an anonymous referee for his very careful reading, and useful comments. 


\section{References}

[1] D. Aharonov and K. Stephenson. Geometric sequences of discs in the Apollonian packing. Algebra i Analiz., 1997. MR1466797

[2] J. Bourgain and E. Fuchs. A proof of the positive density conjecture for integer Apollonian circle packings. Arxiv preprint arXiv:1001.3894, 2010 .

[3] R. Descartes. Oeuvres de Descartes, Correspondance IV (eds. C. Adam and P. Tannery). Leopold Cerf, Paris, 1901.

[4] R. L. Graham, J. C. Lagarias, C. L. Mallows, A. R. Wilks, and C. Yan. Apollonian circle packings: number theory. J. Number Theory 100 (2003), 1-45. MR1971245

[5] R. L. Graham, J. C. Lagarias, C. L. Mallows, A. R. Wilks, and C. Yan. Apollonian circle packings: Geometry and Group Theory I. The Apollonian Group. Discrete and Computational Geometry 34 (2005), 547-585. MR2173929

[6] R. L. Graham, J. C. Lagarias, C. L. Mallows, A. R. Wilks, and C. Yan. Apollonian circle packings: Geometry and Group Theory II. SuperApollonian Group and Integral Packings. Discrete and Computational Geometry 35 (2006), 1-36. MR2183489

[7] R. L. Graham, J. C. Lagarias, C. L. Mallows, A. R. Wilks, and C. Yan. Apollonian circle packings: Geometry and Group Theory III. Higher Dimensions. Discrete and Computational Geometry 35 (2006), 37-72. MR2183490

[8] J. C. Lagarias, C. L. Mallows, and A. R. Wilks. Beyond the Descartes circle theorem. Amer. Math. Monthly 109 (2002), 338-361. MR1903421

[9] J. G. Mauldon. Sets if equally inclined spheres. Canadian J. Math 14 (1962), 509-516. MR0142031

[10] D. Mumford, C. Series, and D. Wright. Indra's Pearls. Cambridge U. P., 2002. MR1913879

[11] S. Northshield. On Apollonian circle packings. Preprint, July 26, 2005.

[12] K. Stephenson. Introduction to circle packing. Cambridge U. P., 2005. MR2131318

[13] J. B. Wilker. Inversive Geometry, in The Geometric Vein (eds. C. Davis, B. Grunbaum and F. A. Sherk), Springer-Verlag, New York, 1981, 379442. MR0661793 
Gerhard Guettler

University of Applied Sciences Giessen Friedberg

Germany

E-mail address: dr.gerhard.guettler@swd-servotech.de

Colin Mallows

AVAYA LABS

BASKING RIDGE, NJ 07920

USA

E-mail address: colinm@research.avayalabs.com

Received January 27, 2010 